\title{
Constraints on spontaneous transfer in problem-solving tasks
}

\author{
BARRY S. STEIN \\ Tennessee Technological University, Cookeville, Tennessee \\ and The University of South Florida, Tampa, Florida
}

and

\section{KERRY R. WAY, SAMMIE E. BENNINGFIELD, and CATHLEEN A. HEDGECOUGH \\ Tennessee Technological University, Cookeville, Tennessee}

\begin{abstract}
In three experiments, we investigated the conditions under which relevant knowledge is spontaneously transferred to problem-solving tasks. Subjects were presented with key concepts that could help them solve problems presented at a later time in the experiment. The key concepts were embedded in statements that had surface structures similar or dissimilar to those of the problems and that emphasized relevant or irrelevant properties of the key concepts for the problemsolving task (contextual relevance). The results indicated that the spontaneous transfer of clue information to subsequent problem-solving tasks is affected by the contextual relevance and the surface-structure similarity of the clue statements to the problems. The findings also suggested that the surface-structure similarity and the contextual relevance of clue statements differentially affect the accessibility and appropriate application of key concepts in problem-solving tasks. The implications of these results for understanding the transfer of knowledge in problem-solving tasks are discussed.
\end{abstract}

Successful problem solvers appear to benefit from both factual knowledge and general problem-solving skills (e.g., Bransford \& Stein, 1984; Greeno, 1980; Simon, 1980). However, the availability of relevant knowledge and general skills is not always sufficient to ensure successful problem solving. As Simon (1980) noted, "The student may possess calculus skills without recognizing that they are applicable to a particular physics problem or without knowing exactly how to apply them" (p. 82). The important role that prior experience plays in comprehension and problem solving ultimately depends on the problem solver's ability to spontaneously access that knowledge at the appropriate time and apply it in a productive way to the new situation.

One method that has been used to investigate the transfer of knowledge in problem-solving tasks is to first present information that is relevant to the solution of a problem and then at some later point in time present the problem (e.g., Perfetto, Bransford, \& Franks, 1983; Weisberg, DiCamillo, \& Phillips, 1978). For example, Perfetto et al. (Experiment 1) presented a list of statements during the initial acquisition stage of the experiment, some of which were clues for problems presented in the second phase of the experiment. Perfetto et al. used

Part of this research was presented at the Southeastern Psychological Association meeting in Atlanta, March 1983. We would like to thank John Bransford, Linda Bettoli, Alice Healy, and Douglas Nelson for their helpful comments on an earlier version of this manuscript.

Barry S. Stein's address is: Psychology Program, Box 5074, Tennessee Technological University, Cookeville, TN 38505. problems such as the following: "A man who lived in a small town in the U.S. married 20 different women of the same town. All are still living and he has never divorced one of them. Yet, he has broken no law. Can you explain?"' The statement that was presented as a clue for this problem was "A minister marries several people each week." Perfetto et al. found that people did not spontaneously transfer the clue information to the problems unless they were specifically informed, prior to the presentation of the problems, about the connection between the clue statements presented earlier in the experiment and the problems (see also Weisberg et al., 1978).

The results reported by Perfetto et al. (1983) and Weisberg et al. (1978) demonstrate a consistent failure to spontaneously transfer information in problem-solving tasks. These findings are particularly significant because, as Perfetto et al. (1983) noted, "Many, if not most, ordinary problem solving situations involve cases in which the problem solver is essentially uninformed [about the knowledge that might be relevant to the task]" (p. 30). If their results are characteristic of performance in these ordinary problem-solving situations, then transfer should seldom occur.

Despite the findings of Perfetto et al. (1983) and Weisberg et al. (1978), it is apparent that people do solve problems in ordinary problem-solving situations without external episodic prompts to help them access relevant knowledge. In fact, experts in any domain may have no one else to rely on to help them access and apply relevant knowledge to a problem. These observations suggest that 
there may be other factors that influence the spontaneous transfer of information in ordinary problem-solving situations.

One factor that has been thought to influence transfer in other experimental paradigms is the similarity between the original learning situation and the transfer task (e.g., McGeoch, 1932; Simon, 1980). For example, investigations of retention demonstrate that the accessibility of acquired information is influenced by the similarity or degree of overlap between information studied during acquisition and retrieval information available at the time of test (e.g., Barclay, Bransford, Franks, McCarrell, \& Nitsch, 1974; Tulving \& Thomson, 1973). The results of investigations such as these suggest that the probability of accessing relevant information in problem-solving tasks may increase as the similarity between the encoding of relevant (clue) information and the encoding of subsequently encountered problem increases.

In order to optimize transfer in ordinary problemsolving situations, successful problem solvers might elaborate key concepts with other information (i.e., retrieval cues) that they would expect to find in a problemsolving situation. For example, the learner could elaborate the encoding of a key concept with words, phrases, or visual images that would be physically present in the problem-solving situation. This strategy of selecting and encoding features of the retrieval environment together with to-be-remembered information is the basis for many mnemonic techniques (e.g., Bransford \& Stein, 1984; Hayes, 1981).

An examination of the clue statements and problems used by Perfetto et al. (1983) reveals little similarity or overlap in features, as reflected by the number of words found in both the clue and problem statements (see example of problem and clue above). Furthermore, since subjects in that study were unaware that they would need to use the information provided during acquisition to solve problems, it is doubtful that they elaborated the clue statements with additional information that would facilitate retrieval on the subsequent problem-solving tasks. As a result, the lack of similarity or overlap in word usage between clues and problems may have been responsible for the subjects' failure to access and apply clue information to the problems.

There may also be factors other than similarity or overlap in word usage that influence the spontaneous transfer of information to problems. For example, Barclay et al. (1974) demonstrated that retrieval cues not present during acquisition (e.g., "something heavy,") could facilitate access to concepts (e.g., "piano") if the acquisition context caused learners to focus on relevant properties of the key concept (e.g., "The man lifted the piano"). Furthermore, such retrieval cues (e.g., "something heavy") were not effective aids to recall if the acquisition context caused learners to focus on irrelevant properties of the key concept (e.g., "The man tuned the piano").
These findings suggest that it may be necessary to emphasize the relevant properties of clue concepts during acquisition in order to facilitate the accessibility of that information during problem solving.

Acquisition contexts that cause learners to focus on relevant properties of key concepts may also improve transfer by facilitating the appropriate application of key concepts to a problem once the information is accessed. For example, the clues used by Weisberg et al. (1978) may have been accessed during problem solving but ignored by subjects because they did not perceive the clues to be relevant to the solution of the problem. In that study, subjects learned paired associates, including the pair "candlebox." This association was expected to cue a solution to the "candle problem" (a variation of Duncker's [1945] "box problem"). The candle problem involves determining how to attach a candle to the wall so that it will burn properly, using a box of nails, a book of matches, a hammer, and the candle. The solution sought by Weisberg et al. for this problem involved emptying the box of nails, attaching the box to the wall with a nail and hammer, and then placing the candle in or on the box. It is questionable whether, in Weisberg et al.'s study, the paired associates "candle-box" prompted subjects to think during the presentation of the paired associates about using a box to attach a candle to the wall. It is more likely that subjects thought about the box as a container (i.e., a box of candles). Consequently, subjects who were uninformed about the relationship between the clues and the problems (spontaneous transfer condition) may have remembered the paired associates "candle-box" while attempting to solve the problem, and yet may not have made the inferences necessary to realize that a candle could be attached to the wall with a box. The failure to appropriately apply the clue information to the problem could be due to the fact that the subjects did not consider relevant properties of the clue concept during the initial presentation of the clue.

In the present series of investigations, we examined the effects of two variables thought to influence the spontaneous transfer of information in problem-solving tasks. As in previous investigations, subjects were first presented with clue statements and then given a set of problems to solve. Subjects were either informed or not informed about the connection between the clues and the problems. In addition, in the present study we simultaneously manipulated the similarity of words used in the clue and problem statements (surface-structure similarity) and the clue statements' emphasis on relevant or irrelevant properties of the key concepts needed to solve the problems (contextual relevance). Problems were selected that could be effectively cued with one- or two-word concepts. These clue concepts were embedded in statements that varied in surface-structure similarity and contextual relevance to the problems. In Experiment 1, the clue statements were presented in an incidental learning task. 


\section{EXPERIMENT 1}

\section{Method}

Subjects. One hundred undergraduate students enrolled in introductory psychology classes at Tennessee Technological University participated for course credit.

Materials. The problems used in the present study were similar to those used by Perfetto et al. (1983) in that subjects were asked to provide plausible explanations for seemingly implausible events. However, the problem statements used here were shorter than those used by Perfetto et al. and were designed so that a one- or twoword concept would prompt a solution to the problem. The five statements that constituted the problem set were adapted from Auble, Franks, and Soraci (1979). The problems and clues are provided in the Appendix. The key concepts used by Auble et al. for clarifying these statements were embedded in four types of acquisition statements (similar surface structure/relevant context [SS/RC], dissimilar surface structure/relevant context [DS/RC], similar surface structure/irrelevant context [SS/IC], and dissimilar surface structure/irrelevant context [DS/IC]). Similar-surface-structure clue statements contained at least two content words that were also found in the problem statements, whereas dissimilar-surface-structure clue statements did not use any content words that were found in the problem statements. Relevant-context statements prompted subjects to consider properties of the key concept that were relevant to the solution of the problem, whereas irrelevant-context statements prompted subjects to consider properties of the key concept that were irrelevant to the solution of the problem. For example, given the problem "The home was small because the sun came out," a clue statement with a relevant context is "An igloo is a home that can be damaged by heat," because the clue statement uses the key word "igloo" in a relationship that emphasizes the deleterious effects of heat on that type of structure. In contrast, a clue statement with an irrelevant context, such as "Some Eskimos live in an igloo," does not use the key word "igloo" in a relationship that emphasizes the deleterious effects of heat on that type of structure. Irrelevant clue statements (i.e., statements that were unrelated to the problems and that did not include the key concepts) were used for the control groups.

In order to verify that the RC clue statements emphasized properties of the key concepts that were more relevant to the problems than the properties emphasized by the IC clue statements, we gave a separate group of 15 undergraduates each problem statement together with the four types of clue statements. These students were asked to rate the similarity in meaning of each clue statement to its respective problem statement, using a 5-point scale with 5 corresponding to the highest degree of similarity. The mean ratings for SS/RC, DS/RC, SS/IC, and DS/IC clue statements were 4.02 , $4.22,1.77$, and 1.14 , respectively. A $2 \times 2$ analysis of variance was performed on the rating scores. There were significant effects for surface-structure similarity $[F(1,14)=10.4, p<.01, M S e=$ $.066]$, for contextual relevance $[F(1,14)=408.8, p<.001$, MSe $=.261]$, and for the interaction between surface structure and contextual relevance $[F(1,14)=20.9, p<.001, M S e=.123]$. The clue statements with relevant contexts (SS/RC and DS/RC) were rated as significantly more similar in meaning to the problem statements than were clue statements with irrelevant contexts (SS/IC and DS/IC) [similar surface structure, $t(42)=15.3$; dissimilar surface structure, $t(42)=21.8$; both $p \mathrm{~s}<.01, M S \mathrm{es}=.15]$. The significant effects for surface-structure similarity and the interaction can be attributed to the fact that SS/IC clue statements were rated as being more related to the problems than were DS/IC clue statements $[t(42)=4.47, p<.01]$. There was no significant difference in ratings between SS/RC and DS/RC clue statements.

Design and Procedure. Subjects were randomly assigned to 1 of 10 experimental conditions in a 2 (relevance instructions) $\times 5$ (clue type) between-groups design and were tested in groups ac- cording to condition. In each experimental condition, subjects were given five clue statements, all of the same type (SS/RC, DS/RC, SS/IC, or DS/IC); subjects in the control conditions were given five irrelevant clue statements. The five clue sentences were embedded in a list with 10 filler sentences that were unrelated to the clues and to the problems. The first and last sentences in the set were always filler sentences. During acquisition, subjects were instructed to rate the truthfulness of the 15 statements, using a 3-point scale ( $1=$ always true, 2 = sometimes true, $3=$ never true $)$. The filler sentences included statements that could be rated 1,2 , and 3 . Subjects were given $20 \mathrm{sec}$ to record their rating for each sentence. After rating the 15 sentences, a period of $30 \mathrm{sec}$ elapsed before the subjects were presented with the five problem statements.

In addition to manipulating the clue context, we informed half of the subjects in each experimental condition about the relevance of the clues for solving problems, and did not inform the other half. Subjects in the informed groups were told that clues for the problem statements they were about to receive may have been present in the list of statements they had previously rated. Subjects in the uninformed groups were not told before solving the problems about the presence of clues in the acquisition list, and thus were led to believe that the two tasks were unrelated. All subjects were given $45 \mathrm{sec}$ to write a solution for each problem statement.

\section{Results}

Two judges independently rated subjects' answers to the problems. The answers were considered correct if they included the key concept (e.g., "igloo") together with some explanation of how that concept was relevant to the solution of the problem. For example, given the problem "The home was small because the sun came out," an answer such as "The home was an igloo and melted" was correct. Answers such as "The angle of the sun made the home seem smaller" or "Some Eskimos live in igloos" were considered incorrect because they did not adequately explain how the key concept ("igloo") was relevant to the solution of the problem. The agreement between judges exceeded $96 \%$. On disputed answers a third opinion was obtained to reach a decision.

The mean percentage of problems solved correctly in each condition is presented in Table 1 . A $2 \times 5$ betweengroups analysis of variance was performed on the percentage of problems solved correctly by each subject $(F)$. An additional $2 \times 5$ analysis of variance was performed to evaluate the stability of effects across items. This analysis $\left(F^{\prime}\right)$ treated items as a random effect. Significant treatment effects were found for clue context $[F(4,90)=$ $\left.34.23, p<.001 ; F^{\prime}(4,40)=19.2, p<.001\right]$ and relevance instructions (informed vs. uninformed) $[F(1,90)$ $\left.=57.47, p<.001 ; F^{\prime}(1,40)=32.2, p<.001\right]$, and the interaction between clue context and relevance instructions was also significant $[F(4,90)=6.04, p<.001$, $\left.M S e=312 ; F^{\prime}(4,40)=3.38, p<.05, M S e=279\right]$.

Several comparisons were performed using Dunn's multiple comparison procedure $\left(t^{\prime}\right)$ to investigate the in-

Table 1

Mean Percentage of Problems Solved in Experiment 1

\begin{tabular}{|c|c|c|c|c|c|}
\hline \multirow{2}{*}{$\begin{array}{l}\text { Relevance } \\
\text { Instructions }\end{array}$} & \multicolumn{5}{|c|}{ Clue Statement } \\
\hline & SS/RC & DS/RC & SS/IC & $\mathrm{DS} / \mathrm{IC}$ & Control \\
\hline Informed & 82.0 & 68.0 & 32.0 & 34.0 & 2.0 \\
\hline Uninformed & 40.0 & 22.0 & 18.0 & 2.0 & 2.0 \\
\hline
\end{tabular}


teraction. To assess the degree of transfer of clues to the problem-solving tasks, we compared performance in each experimental condition with the performance of the control group, which received no clues. In the uninformed conditions, only subjects who received clues embedded in SS/RC statements performed significantly better than the control group $\left[t^{\prime}(90)=4.81, p<.01\right]$, indicating that they spontaneously transferred clue information to the problems. The difference in performance between the control group and those who received DS/RC statements approached significance $\left[t^{\prime}(90)=2.53, p>.05\right]$, as did the difference between the control group and those who received SS/IC statements $\left[t^{\prime}(90)=2.14, p>.05\right]$. It is somewhat difficult to interpret the reliability of the latter effects because of the large error variance in the experiment. However, the results clearly demonstrate that spontaneous transfer can occur, given clue statements with contexts that are relevant and surface structures that are similar to those of the problems.

In the informed conditions, subjects in all experimental conditions performed significantly better than did the control group [for the SS/RC, SS/IC, DS/RC, and DS/IC conditions, respectively, $t^{\prime}(90)=10.13, p<.01 ; t^{\prime}(90)$ $=3.80, p<.01 ; t^{\prime}(90)=8.35, p<.01 ; t^{\prime}(90)=4.05$, $p<.01]$. These results indicate that all clue statements facilitated performance, relative to that of the control group, when subjects were informed about the potential relevance of the clue statements for solving the problems. Statements with relevant contexts (SS/RC and DS/RC) produced significantly better performance than statements with irrelevant contexts (SS/IC and DS/IC) $\left[t^{\prime}(90)=7.51\right.$, $p<.01$ ], indicating that prompting subjects to consider properties of the key concepts that are relevant to the problem-solving task is an important determinant of transfer in the informed conditions. In contrast, no significant difference in problem-solving performance was found between subjects who were given statements with similar surface structure (SS/RC and SS/IC) and those who were given statements without similar surface structure (DS/RC and DS/IC) $\left[t^{\prime}(90)=1.07, p>.05\right]$.

\section{Discussion}

The results of Experiment 1 partially replicate previous findings by demonstrating a failure to spontaneously transfer information encoded in one phase of the experiment to problems presented at a later time in the experiment. However, the results also demonstrate that subjects will spontaneously transfer clue information to a problemsolving task in certain situations. For example, spontaneous transfer did occur when the key concepts were embedded in statements with surface structures similar to those of the problems and those statements emphasized relevant properties of the key concepts.

These results demonstrate conditions that are sufficient to produce the spontaneous transfer of clue information to a problem-solving task. However, it is unclear whether these conditions are necessary for spontaneous transfer to occur. For instance, it could be argued that certain types of clue statements were more difficult to learn than others.
As a result, some of the clue information may never have been available in the subjects' memory. In this case, failure to solve the problems correctly could simply be a result of not having learned the information, rather than of failing to transfer previously acquired information. To evaluate this hypothesis, it is necessary to ensure that the clue information is available in memory before testing for transfer. In Experiment 2, we examined the rate of spontaneous transfer after subjects memorized the clue statements.

\section{EXPERIMENT 2}

\section{Method}

Subjects. One hundred undergraduate students enrolled in introductory psychology classes at Tennessee Technological University participated for course credit.

Materials. The materials were the same as those used in Experiment 1 .

Design and Procedure. The subjects were assigned to 1 of 10 experimental conditions in a 2 (relevance instructions) $\times 5$ (clue type) between-groups design and were tested in groups according to condition. The procedure was similar to that of Experiment 1, except that the clue statements were presented in an intentionallearning task. In each experimental condition, subjects were given five clue statements, all of the same type (SS/RC, DS/RC, SS/IC, or DS/IC); subjects in the control conditions were given five irrelevant clue statements. The five clue statements were embedded in a list with 10 filler sentences. The subjects were asked to study the sentences for $2 \mathrm{~min}$ before a free-recall test, lasting $3 \mathrm{~min}$, was administered. The study-test procedure was repeated until all subjects recalled the sentences verbatim (this required four trials in all conditions). The problems were presented $30 \mathrm{sec}$ after the final recall test. Following acquisition of the clue statements, the procedure was identical to that of Experiment 1.

\section{Results}

Subjects' answers were scored according to the criteria and procedures outlined in Experiment 1. The agreement between judges exceeded $92 \%$.

The mean percentage of problems solved correctly in each condition is presented in Table 2 . A $2 \times 5$ betweengroups analysis of variance was performed on the percentage of problems solved correctly by each subject $(F)$. An additional $2 \times 5$ analysis of variance was performed using items as the random effect $\left(F^{\prime}\right)$. Significant treatment effects were found for clue context $[F(4,90)=$ $\left.49.37, p<.001 ; F^{\prime}(4,40)=40.5, p<.001\right]$ and relevance instructions $[F(1,90)=56.49, p<.001$; $\left.F^{\prime}(1,40)=49.2, p<.001\right]$, and the interaction between clue context and relevance instructions was also significant $\left[F(4,90)=5.10, p<.001, M S e=308 ; F^{\prime}(4,40)\right.$ $=4.22, p<.01$, MSe $=188]$.

Several comparisons were performed using Dunn's multiple comparison procedure $\left(t^{\prime}\right)$ to further investigate

Table 2

Mean Percentage of Problems solved in Experiment 2

\begin{tabular}{lccccc}
\hline \multirow{2}{*}{$\begin{array}{l}\text { Relevance } \\
\text { Instructions }\end{array}$} & \multicolumn{5}{c}{ Clue Statement } \\
\cline { 2 - 6 } & SSC & DS/RC & SS/IC & DS/IC & Control \\
\hline Informed & 82.0 & 78.0 & 32.0 & 20.0 & 4.0 \\
Uninformed & 50.0 & 26.0 & 6.0 & 2.0 & 0 \\
\hline
\end{tabular}


the interaction. In order to assess the degree of transfer, we compared the mean performance in each experimental condition with the performance of the control group, which received no clues. In the uninformed conditions, only subjects who received clues embedded in SS/RC or DS/RC statements performed significantly better than the control group $\left[t^{\prime}(90)=6.37, p<.01\right.$, and $t^{\prime}(90)=3.31$, $p<.05$, respectively], indicating that spontaneous transfer occurred whenever the clue statements prompted subjects to consider relevant properties of the key concepts. Furthermore, the performance of subjects given SS/RC statements was significantly superior to the performance of subjects given DS/RC statements $\left[t^{\prime}(90)=3.06\right.$, $p<.05$ ], indicating that the similarity in surface structure improved spontaneous transfer when the clue statements prompted subjects to consider relevant properties of the key concepts.

In the informed conditions, subjects given SS/RC, SS/IC, and DS/RC clue statements performed significantly better than the control group $\left[t^{\prime}(90)=9.94, p<.01\right.$; $t^{\prime}(90)=3.57, p<.01 ; t^{\prime}(90)=9.43, p<.01$, respectively]. The performance of subjects given clue statements with relevant contexts (SS/RC and DS/RC) was significantly superior to that of subjects given clue statements with irrelevant contexts (SS/IC and DS/IC) $\left[t^{\prime}(90)=9.73\right.$, $p<.01$, indicating that the extent to which clue statements prompt subjects to consider relevant properties of the key concepts is an important determinant of transfer in informed conditions. In contrast, we found no significant difference in transfer between subjects given clue statements with similar surface structures (SS/RC and $\mathrm{SS} / \mathrm{IC}$ ) and those given clue statements with dissimilar surface structures (DS/RC and DS/IC) $\left[t^{\prime}(90)=1.44\right.$, $p>$.05].

\section{Discussion}

The results of Experiment 2 are generally consistent with the data obtained in Experiment 1 . The memorization of clue information did not dramatically alter the rate of spontaneous transfer. These results are consistent with findings reported by Weisberg et al. (1978), and suggest that the ability to retrieve clue information on standard free-recall tests may be unrelated to the probability of spontaneous transfer in a problem-solving task.

As in Experiment 1, the extent to which clue statements prompted subjects to consider relevant properties of the key concepts had an important effect on the degree of spontaneous transfer. Specifically, clue statements with relevant contexts produced spontaneous transfer, whereas clue statements with irrelevant contexts did not produce spontaneous transfer. Furthermore, clue statements with relevant contexts produced spontaneous transfer even when they bore relatively little surface-structure similarity to the problem statements. The latter finding differs from the results of Experiment 1, and suggests that as subjects become more familiar with information, the relevance of the acquisition context has an increasingly important role in spontaneous transfer.
Surface-structure similarity (the use of some identical content words) in clue statements and problem statements appeared to have a less consistent effect on spontaneous transfer. In no case did SS/IC clue statements produce spontaneous transfer. However, SS/RC clue statements did produce more spontaneous transfer than DS/RC clue statements. The latter result suggests that surface-structure similarity may influence spontaneous transfer when subjects have already been prompted to consider relevant properties of the key concepts during acquisition.

One possible explanation for the differential effects of surface-structure similarity and contextual relevance on spontaneous transfer in Experiments 1 and 2 is that there are two processes underlying the spontaneous transfer of information in problem-solving tasks. One process is the accessing of relevant information during the presentation of the problem; the other process is the making of appropriate inferences to correctly apply the accessed information to the problem. In order for spontaneous transfer to occur, both of these activities must take place. Thus, even if the clue information is spontaneously accessed during the problem-solving task, subjects may not solve the problem correctly because they may be unable to make the inferences needed to appropriately apply that information to the problem. Conversely, subjects may be able to appropriately apply the clue information to the problem, but may not solve the problem correctly because of an inability to access that information during the problemsolving task. The effects obtained for surface-structure similarity and contextual relevance in Experiments 1 and 2 may result from the different effects that these variables have on the access and application components of spontaneous transfer.

In Experiment 3, we examined the effects of surfacestructure similarity and contextual relevance on the access and application processes by including a set of control conditions in which the clue and problem statements were presented together. The simultaneous presentation of clues and problems allowed us to evaluate how the clue statements affect the application of key concepts to the problems when the clue information is known to be accessible. Thus, the control conditions allowed us to measure the effects of the clue statements on the appropriate application of clue information in the problem-solving task independently of the effects of clue statements on accessibility. In addition, the number of problems solved correctly in the control conditions provided a baseline for evaluation of changes in the accessibility of clue information when the clue statements were not presented together with the problems (informed and uninformed conditions). That is, by comparing performance in the informed and uninformed conditions with performance in the corresponding control conditions, we could evaluate differences in accessibility independently of the effects of clue statements on the appropriate application of that information to the problem.

In order to further evaluate the effects of clue statements on the access and application components of transfer, we 
included an additional type of clue in Experiment 3. The key concept (e.g., "igloo") was presented alone as a cue for problem solving. The performance of subjects given the key concept alone provided an additional comparison for evaluating the effects of the various clue statements on the access and application of relevant concepts to the problems. Experiment 3 also included a free-recall test so that we could further explore the relationship between the ability to recall clue information and the probability of spontaneous transfer of that information to a problemsolving task.

\section{EXPERIMENT 3}

\section{Method}

Subjects. One hundred ninety-five undergraduate students enrolled in introductory psychology classes at Tennessee Technological University participated for course credit.

Materials. Five types of clue statements were used. Four of these were identical to those used in Experiments 1 and 2: SS/RC, SS/IC, DS/RC, DS/IC. The fifth clue statement consisted of only the key word or concept (e.g., "igloo") needed to solve each problem.

Design and Procedure. Subjects were randomly assigned to one of 15 experimental conditions in a 3 (test condition) $\times 5$ (clue type) between-groups design and were tested in groups according to condition. Subjects in five of the experimental conditions were given the problems simultaneously with the clues (SS/RC, SS/IC, DS/RC, $\mathrm{DS} / \mathrm{IC}$, or key concept alone) and were asked to provide plausible explanations for each problem statement (control condition). The subjects were told that the clue printed next to each problem might contain information that could help them make sense out of the problem statement. These subjects were given $45 \mathrm{sec}$ to solve each problem.

Subjects in the other 10 conditions were given five clues, all of the same type (SS/RC, SS/IC, DS/RC, DS/IC, or key concept alone). The five clues were embedded in a list with 10 filler sentences (or 10 concepts, if the clues were key concepts alone) that were unrelated to the clues or problems. The first and last sentences in the list were always filler sentences. During acquisition, the subjects who received clue statements were instructed to rate the truthfulness of the 15 statements, using a 3-point scale ( 1 = always true, $2=$ sometimes true, $3=$ never true). The filler sentences included statements that could be rated 1,2 , and 3 . Subjects who received the key concepts alone were instructed to rate the list of 15 concepts in terms of pleasantness, using a 3-point scale ( 1 = pleasant, $2=$ neutral, $3=$ unpleasant $)$. The filler items included concepts that could be rated 1,2, and 3 . The subjects were given 2 min to complete the rating task. Thirty seconds after completing this task, they were given a free-recall test lasting $3 \mathrm{~min}$. The problems were presented $30 \mathrm{sec}$ after the final recall test. Subjects in the five informed conditions were told that the information they had rated earlier might contain clues that could help them solve the problems. The subjects in the five uninformed conditions were given no instructions that would lead them to believe that the two tasks were related. All subjects were given $45 \mathrm{sec}$ to solve each problem.

\section{Results}

Subjects' answers for the problems were scored according to the criteria and procedures outlined in Experiment 1 . The agreement between judges exceeded $91 \%$. The mean percentage of problems solved correctly in each condition is presented in Table 3 . An inspection of the means reveals that subjects who were given the clue concepts together with the problem (control groups) solved between $29 \%$ and $91 \%$ of the problems correctly, depend-
Table 3

Mean Percentage of Problems Solved in Experiment 3

\begin{tabular}{lccccc}
\hline \multirow{2}{*}{$\begin{array}{c}\text { Test } \\
\text { Condition }\end{array}$} & SS/RC & DS/RC & SS/IC & DS/IC & Key Concept \\
\cline { 2 - 6 } Control & 86.0 & 90.6 & 29.2 & 60.0 & 75.2 \\
Informed & 64.6 & 61.4 & 16.8 & 12.2 & 41.4 \\
Uninformed & 49.2 & 33.8 & 3.0 & 1.4 & 13.8 \\
\hline
\end{tabular}

ing on the type of clue statement. Even when subjects received the key concepts in SS/RC statements, they failed to solve an average of $14 \%$ of the problems correctly. For example, given the problem statement "The haystack was important because the cloth ripped"' and the clue "A haystack could be helpful if one's parachute ripped," one subject gave the incorrect answer "The hay was helpful to repair the cloth." This observation suggests that even in the SS/RC condition, subjects must make additional inferences in order to solve the problems correctly.

A $3 \times 5$ between-groups analysis of variance was performed on the percentage of problems solved correctly by each subject $(F)$. An additional $3 \times 5$ analysis of variance was performed using items as a random effect $\left(F^{\prime}\right)$. Significant treatment effects were found for type of clue statement $\left[F(4,180)=30.4, p<.001 ; F^{\prime}(4,60)=36.9\right.$, $p<.001]$ and test condition (control vs. informed vs. uninformed) $[F(2,180)=60.2, p<.001, M S \mathrm{e}=631$; $\left.F^{\prime}(2,60)=73.3, p<.001, M S e=341\right]$.

An inspection of the means for the control conditions shown in Table 3 reveals some substantial differences in problem-solving performance when the different clue statements were presented simultaneously with the problems. Although clue statements with relevant contexts (SS/RC, DS/RC) produced the highest levels of performance, they did not produce significantly more correct answers than the key concept alone $\left[t^{\prime}(180)=1.54\right.$, $p>.05]$. However, clue statements with irrelevant contexts (SS/IC, DS/IC) produced significantly fewer correct answers than the key concept alone $\left[t^{\prime}(180)=3.59\right.$, $p<.05]$, indicating that clue statements that prompt subjects to consider irrelevant properties of the key concepts can interfere with the appropriate application of those concepts to the problems. Furthermore, SS/IC clue statements produced significantly fewer correct answers than DS/IC clue statements $\left[t^{\prime}(180)=4.25, p<.01\right]$, indicating that the SS/IC clue statements made it more difficult to appropriately apply the key concepts than did the DS/IC clue statements.

The control groups provided an estimate of subjects' ability to appropriately apply the clue statements to the problems when the clue information was known to be accessible. We compared these performance levels with problem-solving rates in the uninformed conditions to evaluate potential differences (among groups given different types of clue statements) in the accessibility of clue information during problem solving. Subjects given clue statements with similar surface structures (SS/RC, SS/IC) solved an average of $31.5 \%$ fewer problems than did those in the corresponding control conditions, whereas subjects given clue statements with dissimilar surface structures 
(DS/RC, DS/IC) solved an average of $57.7 \%$ fewer problems than did those in corresponding control conditions. Subjects given key concepts alone solved an average of $61.4 \%$ fewer problems than did those in the corresponding control condition, a decrement that was slightly larger than that observed for clues with dissimilar surface structures. In comparison, subjects given clue contexts with similar surface structures (SS/RC, SS/IC) showed a significantly smaller decrease in performance (relative to that of the control group) than subjects given clue contexts with dissimilar surface structures (DS/RC, DS/IC) $\left[t^{\prime}(180)=2.66, p<.05\right]$, indicating that similar surface structure facilitates the accessibility of clue information during problem solving. In contrast, there was not a consistent effect for differences in contextual relevance on accessibility in the uninformed conditions. Subjects given clues with relevant contexts (SS/RC, DS/RC) and subjects given clues with irrelevant contexts (SS/IC, DS/IC) showed comparable performance decrements relative to performance in the corresponding control conditions (46.8\% and $42.4 \%$, respectively).

Additional analyses were performed to determine whether the clue contexts might also affect the accessibility of clue information in the informed conditions. Subjects given clue statements with similar surface structures (SS/RC, SS/IC) solved an average of $16.9 \%$ fewer problems than did those in the corresponding control conditions, whereas subjects given clue statements with dissimilar surface structures (DS/RC, DS/IC) solved an average of $38.6 \%$ fewer problems than did those in the corresponding control conditions. Subjects given key concepts alone solved an average of $33.8 \%$ fewer problems than did those in the corresponding control condition, a decrement that was slightly less than that observed for clues with dissimilar surface structures. The difference in performance decrements between subjects given clues with similar surface structures and those given clues with dissimilar surface structures approached significance $\left[t^{\prime}(180)=2.19, p>.05\right]$. Differences in contextual relevance had little effect on accessibility in the informed conditions. Subjects given clues with relevant contexts (SS/RC, DS/RC) and subjects given clues with irrelevant contexts (SS/IC, DS/IC) showed comparable performance decrements, relative to performance in the corresponding control conditions ( $25.3 \%$ and $30.1 \%$, respectively).

An analysis of the recall scores for subjects in the informed and uninformed conditions was performed to determine if problem-solving performance was related to performance on the free-recall test. Two judges independently scored subjects' responses on the recall test. The responses were considered correct if they included the key concept together with an appropriate paraphrase of the clue statement. The agreement between judges exceeded $97 \%$.

The probability of correctly solving problems given that the clue statement was recalled (CY) and the probability of correctly solving problems given that the clue statement was not recalled $(\mathrm{CN})$ were calculated for each subject. A 2 (CY vs. $\mathrm{CN}) \times 2$ (informed vs. uninformed) $\times$
5 (clue type) split-plot analysis of variance was performed on the percentage of problems correctly solved. Significant treatment effects were found for relevance instructions $[F(1,120)=16.6, p<.001]$, clue condition $[F(4,120)=$ $18.3, p<.001, M S e=1,524]$ and conditional recall (CY vs. $\mathrm{CN})[F(1,120)=4.00, p<.05]$, and the interaction between relevance instructions and conditional recall was also significant $[F(1,120)=6.25, p<.05, M S e=509]$.

The interaction between relevance instructions and conditional recall was due to the fact that subjects in the informed conditions solved a greater percentage of problems for the clues that they recalled than for clues they did not recall $\left(45.9 \%\right.$ vs. $33.3 \%$, respectively) $\left[t^{\prime}(120)=3.18\right.$, $p<.05]$. In contrast, there was not a significant difference in the uninformed condition between the percentage of problems solved for clues that were recalled and those solved for clues that were not recalled $(19.2 \%$ vs. $20.6 \%$, respectively). Further analysis revealed that in the informed conditions, significantly more problems were solved for $\mathrm{CY}$ clues than $\mathrm{CN}$ clues by subjects given clues with relevant contexts (SS/RC and DS/RC) $\left[t^{\prime}(120)=\right.$ $3.79, p<.01]$. However, no significant differences were found between $\mathrm{CY}$ and $\mathrm{CN}$ clues in problems solved by subjects given clues with irrelevant contexts (SS/IC and $\mathrm{DS} / \mathrm{IC})\left[t^{\prime}(120)=0.21, p>.05\right]$, indicating that problem-solving performance in the informed conditions was facilitated by recall of the clue only when the clue context prompted subjects to consider relevant properties of the key concept. The relationship between recall and problem solving in the informed (and uninformed) conditions is summarized in Table 4.

As in Experiments 1 and 2, the group showing the greatest amount of spontaneous transfer (in the uninformed conditions) received SS/RC clue statements $(49.2 \%)$. The performance of subjects in this group was significantly superior to the performance of subjects given the key concept alone $\left[t^{\prime}(180)=3.59, p<.01\right]$, indicating that the SS/RC sentence frame facilitated the spontaneous transfer of the key concept. The difference in performance between subjects in the DS/RC condition and subjects given the key concept alone approached significance $\left[t^{\prime}(180)=2.03, p>.05\right]$.

\section{Discussion}

The results of Experiment 3 suggest that two processes are involved in the spontaneous transfer of information. One of these is the spontaneous accessing of relevant in-

Table 4

Mean Percentage of Problems Solved as a Function of Clue Recall and Clue Statement Condition

\begin{tabular}{lllrr}
\hline \multicolumn{5}{c}{ Clue Recall and Clue Statement Condition } \\
\cline { 2 - 5 } Clue Recall & SS/RC & DS/RC & SS/IC & DS/IC \\
\hline \multicolumn{5}{c}{ Informed Conditions } \\
Yes & 78.2 & 76.9 & 9.6 & 14.7 \\
No & 55.8 & 51.9 & 20.5 & 6.5 \\
& Uninformed Conditions & & \\
Yes & 39.7 & 38.5 & 2.5 & 0 \\
No & 53.2 & 32.0 & 2.5 & 1.9 \\
\hline
\end{tabular}


formation during problem solving; the other is the appropriate application of that information to the problemsolving task. Subjects in the control groups, who were given the key concepts simultaneously with the problems (ensuring accessibility), performed quite differently depending on the type of statement that accompanied the key concept. Specifically, subjects solved the greatest number of problems correctly when the key concepts were presented in statements with relevant contexts, and they solved the fewest problems correctly when the key concepts were presented in statements with irrelevant contexts. Subjects given clue statements with irrelevant contexts produced significantly fewer solutions to the problems than subjects given the key concepts alone. In contrast, similarity in surface structure between clue statements and problems did not facilitate problem-solving performance in the control conditions. These findings suggest that the appropriate application of key concepts to a problem-solving task is primarily influenced by whether the acquisition statements prompt subjects to consider properties of the key concepts that are relevant to the problem-solving task.

To evaluate differences in the accessibility of clue information (when the clues were presented before the problems), we compared the performance of subjects in the informed and uninformed conditions with the performance of subjects in the control conditions. These comparisons provided a means of assessing differences in the accessibility of clue information while taking into consideration the effects of the clue statements on the appropriate application of that information. Problem-solving performance in both the informed and uninformed conditions decreased least (relative to performance in the control conditions) when clue statements had surface structures similar to those of the problems, and decreased most (relative to performance in the control conditions) when clue statements had surface structures dissimilar to those of the problems. In contrast, differences in the contextual relevance of the clue statements appeared to have a less consistent effect on accessibility in the informed and uninformed conditions. These findings suggest that the accessibility of relevant clue information during problemsolving tasks is primarily influenced by similarity in surface structure (i.e., the use of identical content words) in the clue and problem statements.

SS/RC clue statements enhanced both the accessibility of clue information and its appropriate application to the problem-solving tasks. These clue statements also produced significantly more spontaneous transfer in Experiment 3 than did the key concepts alone. It is not surprising that SS/RC clue statements in Experiments 1 and 2 produced spontaneous transfer. The fact that DS/RC clue statements produced spontaneous transfer in Experiment 2 illustrates the relative importance of factors that affect spontaneous transfer. Clues embedded in DS/RC statements may produce spontaneous transfer because the reduced accessibility of clue information is offset by the high probability of deriving an answer once the clue in- formation is accessed. In contrast, clues embedded in SS/IC statements may not produce spontaneous transfer because the reduced probability of appropriate application of the clue to the problem is not offset by a higher probability of that clue's being spontaneously accessed during problem solving.

The relationship between subjects' ability to recall a clue statement prior to the problem-solving task and their ability to solve the problem is less clear. The results indicate that the ability to free-recall clue information was associated with problem-solving performance only in the informed conditions. In fact, the positive relationship between recall of a clue and solution of the corresponding problem in the informed conditions was observed only when subjects were given clues with relevant contexts. One possible explanation for these results is that subjects in the informed conditions employed a strategy of trying to generate each previously presented statement while working on the problems. Clues which were easily remembered in a free-recall test would also have a high probability of being generated during the problem-solving task. Furthermore, clue statements with relevant contexts were more likely to produce correct solutions when they were accessed than were clue statements with irrelevant contexts. Therefore, the advantages of being able to recall a clue statement should be greatest for clues with relevant contexts in the informed conditions. Subjects in the uninformed conditions would not be expected to use a generation strategy because they were not informed that the clue statements presented earlier could help them solve the problems.

The fact that the spontaneous transfer of information in problem-solving tasks (in the uninformed conditions) was not strongly influenced by the ability to free-recall potential clue information in Experiments 2 and 3 is consistent with the findings of Weisberg et al. (1978). The results of the present study suggest that the spontaneous transfer of clue information is determined primarily by the clue statements' contextual relevance and surfacestructure similarity to the problems.

\section{GENERAL DISCUSSION}

The results of the present study help clarify some of the constraints on the spontaneous transfer of information in problem-solving tasks. The findings demonstrate that spontaneous transfer of information in problemsolving tasks is affected by the characteristics of the acquisition context (clue statement) in which key concepts are initially presented. The similarity in surface structure between the acquisition context and the problem statement appears to influence the accessibility of information during problem solving. The extent to which the acquisition context prompts learners to consider properties of the key concepts that are relevant to the solution of the problem (contextual relevance) appears to influence the appropriate application of accessible information to the problemsolving task. 
The findings reported here may also help clarify why spontaneous transfer was not observed in previous investigations of problem solving. For example, the clues and problems used by Perfetto et al. (1983) (see example in introduction) appear to have little surface-structure similarity as reflected by the number of content words used in both clue and problem statements. The present research indicates that a lack of surface-structure similarity between clues and problems could greatly reduce the probability that the clue statements would be spontaneously accessed during the problem-solving task. The failure to spontaneously access relevant clue information during problem solving would certainly make spontaneous transfer difficult. The present findings suggest that spontaneous transfer could have been enhanced if Perfetto et al. had used clue statements with wording more similar to that of the problem statements (e.g., "The minister was a man who married 20 different women of the same town").

Previous research by Weisberg et al. (1978) also failed to find evidence of spontaneous transfer. The clues and problems used in that study appear to satisfy the criterion of surface-structure similarity (see example in introduction). However, in that study it is not clear whether the acquisition context prompted subjects to consider properties of the clue concepts that were relevant to the solution of the problem. The present findings suggest that subjects may have spontaneously accessed the clue concepts during problem solving, but failed to appropriately apply the information to the problem and consequently did not show evidence of spontaneous transfer.

Although the results presented here and in previous studies of spontaneous transfer indicate that people can have considerable difficulty in spontaneously transferring clue information to novel problems, there are clearly ways to optimize that transfer. For instance, one implication of this study is that the probability of spontaneously transferring clue information to problem-solving tasks can be substantially affected by the acquisition context in which key concepts are initially studied. Acquisition contexts that are similar in surface structure to the problem-solving tasks will enhance the probability of spontaneously accessing relevant information during problem solving, and acquisition contexts that emphasize relevant properties of the key concepts will enhance the application of that information to the problem-solving task.

In ordinary learning and problem-solving situations, successful learners and problem solvers may prepare themselves for transfer by trying to anticipate situations in which the information they are studying would be significant and helpful (see also Bransford \& Stein, 1984; Stein et al., 1982). These elaborations could enhance the similarity in surface structure between the acquisition contexts for key concepts and future problem-solving tasks. Furthermore, by trying to anticipate situations in which key concepts might be useful and significant, learners would implicitly be prompting themselves to consider properties of the key concepts that would be relevant to future problem-solving tasks. Therefore, these activities should not only facilitate the accessibility of information in ordinary problem-solving situations, but should also increase the probability that the information would be appropriately applied to the problem.

\section{REFERENCES}

Auble, P. M., Franks, J. J., \& Soraci, S. A. (1979). Effort toward comprehension: Elaboration or "aha"? Memory \& Cognition, 7, 426-434.

Barclay, J. R., Bransford, J. D., Franks, J. J., McCarrell, N. S., \& Nitsch, K. E. (1974). Comprehension and semantic flexibility. Journal of Verbal Learning \& Verbal Behavior, 13, 471-481.

BRANSFORD, J. D., STEIN, B. S. (1984). The ideal problem solver: A guide for improving thinking, learning, and creativity. New York: Freeman.

DUNCKER, K. (1945). On problem solving. Psychological Monographs, $\mathbf{5 8}, 270$.

Greeno, J. G. (1980). Trends in the theory of knowledge for problem solving. In D. T. Tuma \& F. Reif (Eds.), Problem solving and education: Issues in teaching and research. Hillsdale, NJ: Erlbaum.

HAYES, J. R. (1981). The complete problem solver. Philadelphia: Franklin Institute Press.

McGeoch, J. A. (1932). Forgetting and the law of disuse. Psychological Review, 39, 352-370.

Perfetto, G. A., Bransford, J. D., \& Franks, J. J. (1983). Constraints on access in a problem solving context. Memory \& Cognition, 11, 24-31.

SimoN, H. A. (1980). Problem solving and education. In D. T. Tuma \& F. Reif (Eds.), Problem solving and education: Issues in teaching and research. Hillsdale, NJ: Erlbaum.

Stein, B. S., Bransford, J. D., Franks, J. J., Owings, R. A., Vye, N. J., McGRaw, W. (1982). Differences in the precision of selfgenerated elaborations. Journal of Experimental Psychology: General, 111, 399-405.

Tulving, E., \& Thomson, D. M. (1973). Encoding specificity and retrieval processes in episodic memory. Psychological Review, 80, 352-373.

Weisberg, R., DiCamillo, M., \& Phillips, D. (1978). Transferring old associations to new situations: A nonautomatic process. Joumal of Verbal Learning \& Verbal Behavior, 17, 219-228.

\section{APPENDIX \\ Problems and Clue Statements}

Problem: "The home was small because the sun came out." Key Concept: "igloo"

SS/RC Clue Statement: "An igloo is a home that can be damaged by the sun."

DS/RC Clue Statement: "An igloo can be damaged by heat."

SS/IC Clue Statement: "After being out in the sun, my home feels like an igloo."

DS/IC Clue Statement: "Some Eskimos live in an igloo." Irrelevant Clue Statement: "Airplanes are safer than automobiles."

Problem: "The clothes were ruined because the sign vanished."

Key Concept: "wet paint"

SS/RC Clue Statement: "A wet paint sign can prevent people from ruining their clothes."

DS/RC Clue Statement: "A wet paint notice can prevent people from staining their garments."

SS/IC Clue Statement: "Ruined clothes can be used to wipe wet paint off of a sign.",

DS/IC Clue Statement: "Wet paint has a strong smell."

Irrelevant Clue Statement: "All houses should have smoke alarms." 


\section{Appendix (Continued)}

Problem: "The haystack was important because the cloth ripped."

Key Concept: "parachute"

SS/RC Clue Statement: "A haystack could be helpful if one's parachute ripped."

DS/RC Clue Statement: "A pile of straw could be helpful if one's parachute tears."

SS/IC Clue Statement: "A parachute can be ripped by a needle in a haystack.

DS/IC Clue Statement: "The parachute had a bright red color."

Irrelevant Clue Statement: "Newspaper editorials can be biased."

Problem: "The family asked for rooms because the light had burned out."

Key Concept: "no vacancy"

SS/RC Clue Statement: "People will not stop for rooms if a no vacancy sign is lighted."
DS/RC Clue Statement: "People will not stop for accommodations if a no vacancy sign is on."

SS/IC Clue Statement: "People cannot sleep if the light from a no vacancy sign is shining in their room."

DS/IC Clue Statement: "The no vacancy sign was small." Irrelevant Clue Statement: "Dogs make good pets."

Problem: "The notes were sour because the seam split."

Key Concept: "bagpipes"

SS/RC Clue Statement: "The notes were off key because the seams on the bagpipe opened."

DS/RC Clue Statement: "A tear in the bagpipes made the music sound strange."

SS/IC Clue Statement: "The weak seam split when the bagpipe played high notes."

DS/IC Clue Statement: "Bagpipes originated in Scotland." Irrelevant Clue Statement: "Bats fly at night."

\footnotetext{
Manuscript received January 21, 1985 ;

revision accepted for publication February 17, 1986.)
} 